\title{
ESTIMAÇÃO DOS PARÂMETROS E MAPEAMENTO DA FUNÇÃO OBJETIVO PARA MODELO DA COPOLIMERIZAÇÃO DO ETILENO ATRAVÉS DO PSO
}

\author{
I. S. C. BERNARDES ${ }^{1}$, I. B. R. NOGUEIRA ${ }^{2}$ e K. V. PONTES ${ }^{3}$ \\ 1,2,3 Universidade Federal da Bahia, Departamento de Engenharia Química \\ E-mail para contato: karenpontes@ufba.br
}

\begin{abstract}
RESUMO - A estimação de parâmetros constitui uma das etapas do desenvolvimento de um modelo matemático, sendo essencial para a validação e uso do mesmo. Modelos nãolineares, como o presente caso, não permitem solução analítica para a minimização da função objetivo. É preciso usar métodos numéricos iterativos, como os métodos heurísticos. Estes lidam bem com problemas com muitos parâmetros e função objetivo não-suave, descontínua, com mínimos locais. A Otimização por Enxame de Partículas (PSO - Particle Swarm Optimization) foi usada para efetuar o mapeamento da função objetivo para os pares de parâmetros de um modelo fenomenológico do processo de copolimerização do etileno em solução. Os resultados mostram que o PSO foi capaz de encontrar valores ótimos para as estimativas dos parâmetros permitindo a validação e aplicação do modelo.
\end{abstract}

\section{INTRODUÇÃO}

Os fenômenos observáveis são explicados quantitativamente e/ou qualitativamente por modelos científicos. Segundo Pinto e Schwaab (2007), um modelo matemático indica a relação entre variáveis de maneira quantitativa através de entes chamados parâmetros, portanto a estimação de parâmetros é uma etapa fundamental na definição completa de tais modelos e é seguida pela validação do mesmo. Para solucionar tal problema de otimização, algoritmos probabilísticos apresentam algumas seguintes vantagens frente aos determinísticos (HOLTZ, 2005; PINTO e SCHWAAB, 2007):

- Empregam a função objetivo e as restrições com ou sem uma representação matemática;

- Funcionam sem a necessidade de função objetivo contínua ou diferenciável ou de formulações complexas ou reformulações para o problema;

- Trabalham adequadamente, tanto com parâmetros contínuos quanto com discretos, ou ainda com uma combinação deles;

- Realizam buscas simultâneas no espaço de possíveis soluções através de uma população de indivíduos sem quaisquer restrições quanto ao ponto de partida dentro do espaço de busca da solução;

- Otimizam um grande número de variáveis, desde que a avaliação da função objetivo não tenha um custo computacional demasiadamente alto;

- Apresentam equações simples e robustez do procedimento de busca, graças à natureza global e aleatória da pesquisa. 


\section{9 a 22 de outubro de 2014 \\ Florianópolis/SC}

Entre os métodos heurísticos existentes destaca-se a Otimização por Enxame de Partículas (Particle Swarm Optimization - PSO) proposto por Kennedy e Eberhart (1995). Este método se baseia na simulação do comportamento social de animais como cardumes de peixes e bandos de pássaros. No PSO a influência da escolha da estimativa inicial é pequena, permitindo-o fugir de mínimas locais mais facilmente (SCHWAAB et al., 2008). O método também pode ser aplicado em problemas com função objetivo não-suave (PARK et al., 2005). Poli (2008) sugere ao menos 26 áreas de pesquisa nas quais o uso do PSO ainda se mostra muito fértil. São assuntos que passam pela Biomedicina, Modelagem, Otimização e Projeto e chegam até em usos para lógica Fuzzy e Redes Neurais. Schwaab (2005) aponta o PSO como o método mais adequado para problemas de estimação de parâmetros.

O objetivo deste trabalho é estimar os parâmetros do modelo fenomenológico da copolimerização do etileno com 1-buteno em uma série de reatores (PONTES et al., 2010). Tendo em vista o elevado número de parâmetros a ser estimado e às vantagens citadas, o PSO é utilizado para solução do problema de otimização, permitindo também o mapeamento da função objetivo. Para a estimação foi utilizada uma série de dados amostrais de uma planta industrial.

\section{METODOLOGIA}

O modelo cujos parâmetros são o objeto de estudo deste trabalho foi desenvolvido por Pontes $e t$ al (2010) e descreve a copolimerização de etileno e 1-buteno em solução com catalisador ZieglerNatta. O processo ocorre ao longo de um conjunto de reatores dos tipos tanque agitado contínuo (Continuous Stirred Tank Reactor - CSTR) e tubular (Plug Flow Reactor - PFR). O catalisador é baseado em uma mistura de titânio e vanádio ativada por trietil alumínio, antes da polimerização. $\mathrm{O}$ mesmo é alimentado em sua forma ativada, pois o processo de ativação ocorre em um tanque de alimentação do catalisador. O sistema é adiabático e não faz uso de sistemas de refrigeração (PONTES et al., 2010). A Figura 1 mostra o fluxograma do processo.

O modelo é descrito por um conjunto de equações diferenciais que apresenta não-linearidades que o tornam bastante complexo. Dentre as variáveis preditas estão: temperatura do topo do CSTR; temperatura da base do CSTR; temperatura de saída do $\mathrm{PFR}_{2}$; produtividade. Estas variáveis foram normalizadas por questão de confidencialidade dos dados industriais. Para que o modelo seja válido com as condições de operação industrial, é necessário estimar os parâmetros, dentre eles energias de ativação e fatores de Arrhenius para cálculo das constantes cinéticas, calores específicos à pressão constante e parâmetros de misturas. Foi realizada previamente uma análise de estimabilidade dos parâmetros (BESSA e PONTES, 2014) que restringiu o número de parâmetros a estimar a um total de 24 dentre os 69 iniciais. 


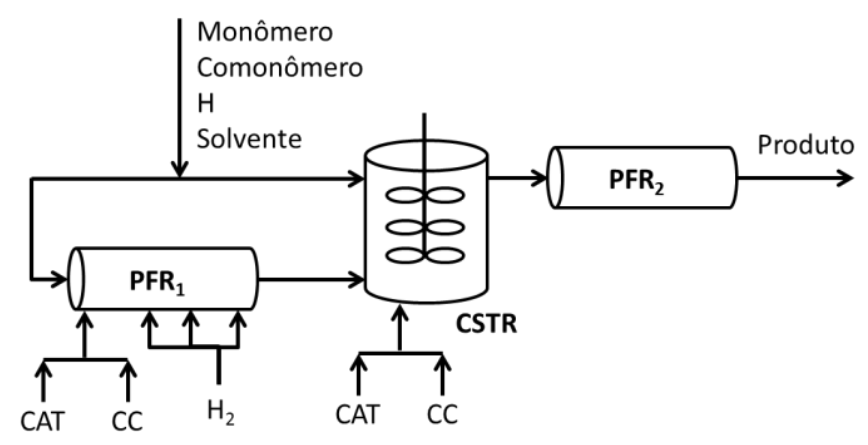

Figura 1 - Fluxograma de processo da copolimerização.

A estimação é efetuada minimizando o logaritmo natural da soma dos quadrados das diferenças entre os valores experimentais e os preditos pelo modelo. Essa métrica foi usada por Benyahia et al. (2010) e Benyahia et al. (2013) na estimação de parâmetros químicos de uma reação de polimerização de um modelo com múltiplas variáveis de saída. Seu uso é conveniente por sua versatilidade, já que permite a avaliação de várias variáveis preditas simultaneamente e com diferentes números de medidas experimentais associadas a cada uma dessas variáveis. A função objetivo é dada por:

$J(P)=\sum_{i=1}^{n_{y}} n_{m i} \ln \left(\sum_{j=1}^{n_{m i}}\left(y_{i}^{e x p}\left(t_{i j}\right)-y_{i}^{\text {pred }}\left(X, P, t_{i j}\right)\right)^{2}\right)$

onde $J(P)$ é a função do critério de verossimilhança $J, n_{y}$ é o número de variáveis preditas pelo modelo, $n_{m i}$ é o número de medidas experimentais de cada i-ésima variável de saída, $t_{i j}$ é o j-ésimo tempo de medição da variável $y_{i}, y_{i}^{\text {pred }}$ é o valor predito da variável $y_{i}$ definidas as variáveis preditoras $X$, os parâmetros estimados $P$ e o tempo $t_{i j}$.

A variante do PSO empregada no presente trabalho foi o Enxame de Partículas hierárquico auto organizado com coeficientes de aceleração variando com o tempo ou Self-Organizing Hierarchical Particle Swarm Optimizer With Time-Varying Acceleration Coefficients (HPSO-TVAC). Cada partícula carrega os valores de estimativa dos parâmetros e tem sua velocidade $(v)$ e posição $(x)$ atualizadas a cada iteração, de acordo com:

$v_{p, d}^{k+1}=v_{p, d}^{k}+c_{1} r_{1}\left(x_{p, d}^{i n d}-x_{p, d}^{k}\right)+c_{2} r_{2}\left(x_{d}^{g l o}-x_{p, d}^{k}\right)$

$x_{p, d}^{k+1}=x_{p, d}^{k}+v_{p, d}^{k+1}$

onde $k$ é o número da iteração, $p$ representa a partícula, $d$ é a direção de busca, ou seja, um dos parâmetros, $x^{\text {ind }}$ é a melhor posição histórica da partícula, $x^{g l o}$ é a melhor posição histórica de todo o conjunto de partículas, $c_{1}$ e $c_{2}$ são os parâmetros cognitivo e social de busca e $r_{1}$ e $r_{2}$ são número aleatórios tomados de um intervalo de [0,1] com distribuição uniforme. Os parâmetros cognitivo $\left(c_{1}\right)$ e social $\left(c_{2}\right)$ são usados para balancear o caráter local e global da busca. 


\section{9 a 22 de outubro de 2014 \\ Florianópolis/SC}

Valores maiores de $c_{1}$ fazem com que as partículas façam buscas mais limitadas às proximidades de seu mínimo histórico. Valores mais altos de $c_{2}$ aumentam a interação entre as partículas levando-as a intensificar as pesquisas nas vizinhanças do mínimo global. Ratnaweera et al (2004) propuseram o HPSO-TVAC, o qual apresenta a propriedade especial de reinicializar as partículas dando-lhes velocidades aleatórias sempre que elas estagnarem no espaço de busca. Isso aumenta a exploração do espaço. O algoritmo apresentado por Schwaab et al. (2008) e adaptado para a variante HPSO-TVAC conforme apresentado no pseudocódigo por Ratnaweera et al (2004) foi aplicado utilizando as condições definidas na Tabela 1 , onde $n_{p}$ representa o número de parâmetros, $n_{y}$ é o número de variáveis de saída do modelo, $n_{m}$ é o total de medidas experimentais de todas as variáveis, Nit é o número de iterações empregadas, $N p t$ é o número de partículas usadas na busca, $N d$ é o número de dimensões de busca e é igual ao número de parâmetros que estão sendo estimados e $X^{M I N}$ e $X^{M A X}$ são vetores com os valores mínimos e máximos aceitáveis para os parâmetros durante a busca. Os limites de busca foram inicialmente estabelecidos com base em trabalhos anteriores realizados pelo grupo (EMBIRUÇU et al., 2008; PONTES et al, 2010). Os limites de busca encontrados na Tabela 1 estão normalizados de forma a melhorar a convergência do algoritmo.

Tabela 1 - Condições do algoritmo e do problema

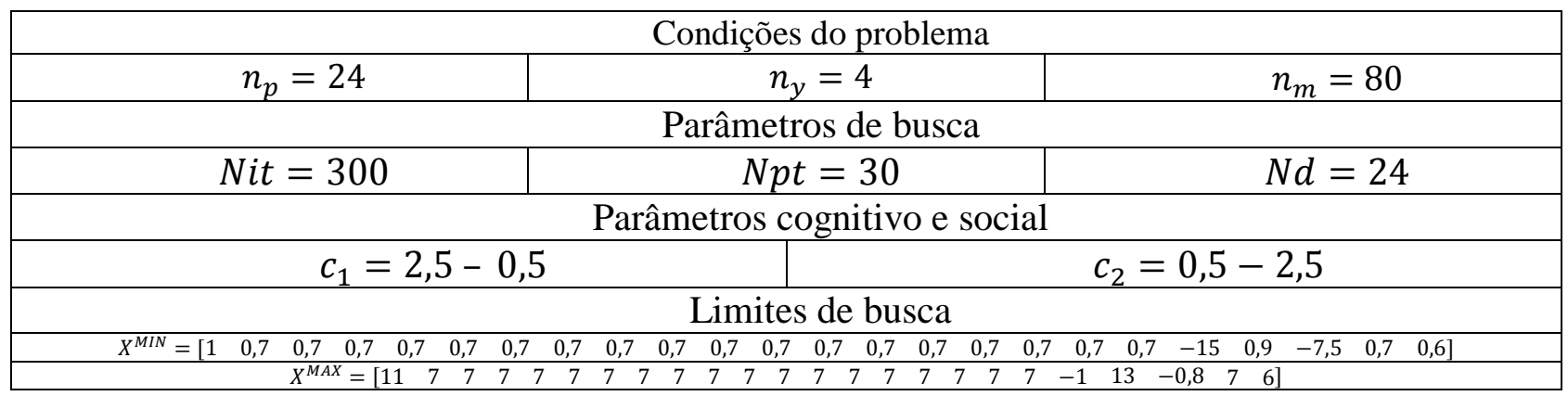

O mapeamento da função objetivo é importante para verificar o comportamento da função objetivo em relação aos parâmetros. Essa informação é importante para avaliar a qualidade da estimação e das estimativas iniciais. O mapeamento foi efetuado plotando o valor da função objetivo em função de cada par de parâmetros, usando todas as posições históricas das partículas do enxame.

O algoritmo do PSO foi implementado em Matlab e o modelo do processo em Fortran, sendo necessária comunicação entre os ambientes.

\section{RESULTADOS E DISCUSSÃO}

O tempo de execução do algoritmo foi cerca de 12 horas em um computador com 4GB de memória, processador Qemu Virtual CPU Version (CPU64 - RHEL6) 2,39GHz e sistema operacional Windows Server 2008 R2 Enterprise - Service Pack 1. Dos 24 parâmetros estimados, 14 tiveram valor ótimo próximo aos limites de busca. Um indicativo de que há a possibilidade de ainda serem encontradas condições melhores para o conjunto de parâmetros. 
As Figuras de 2 a 5 exibem os valores preditos pelo modelo ajustado e os valores de um conjunto amostral. Todos os valores estão normalizados por confidencialidade industrial. Nota-se que o modelo prediz bem os valores experimentais da produtividade. $\mathrm{O}$ erro máximo encontrado não ultrapassa 2,4\% em relação ao valor medido. A dinâmica da mesma também é bem seguida, tanto no decréscimo de aproximadamente $7,2 \%$ quanto no incremento próximo aos 9,0\%. A temperatura da base do CSTR tem boa qualidade de predição nos instantes iniciais, com erros em torno de 0,37\%, e, à medida em que o valor experimental é incrementado, o desvio chega próximo de $3,8 \%, \log$ a ainda pequeno. No instante de amostragem próximo de 50 a temperatura de saída do processo sofre um incremento em torno de 2,8\%. A temperatura do topo do CSTR tem um incremento de 2,3\% ao longo da amostragem. No início a diferença entre o previsto e o experimental é de 6,9\%, mas essa diferença diminui até cerca de $1,2 \%$.

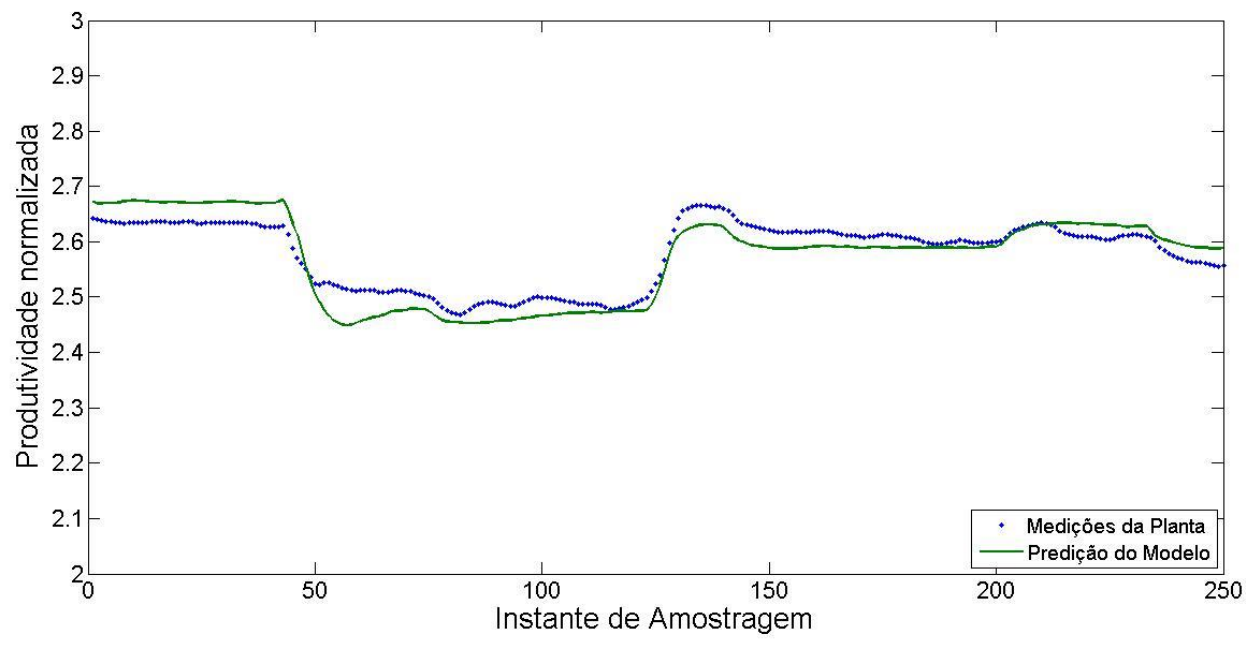

Figura 2 - Valores preditos e experimentais: Produtividade normalizada.

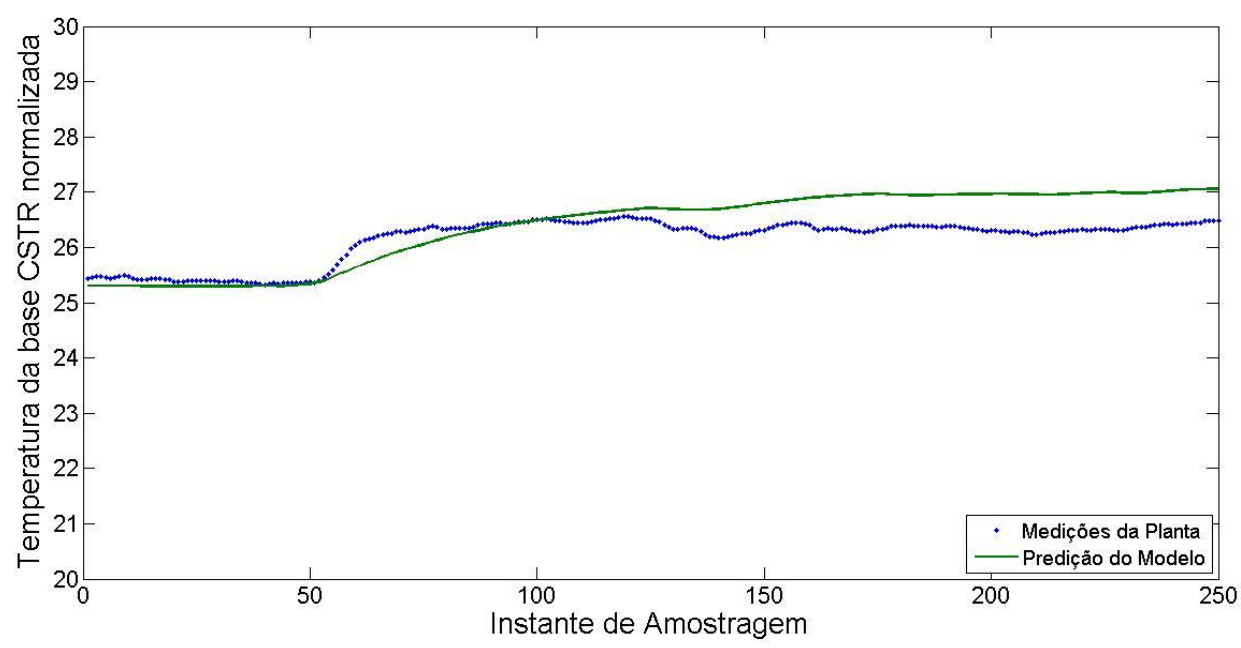

Figura 3 - Valores preditos e experimentais: Temperatura da base do CSTR normalizada. 


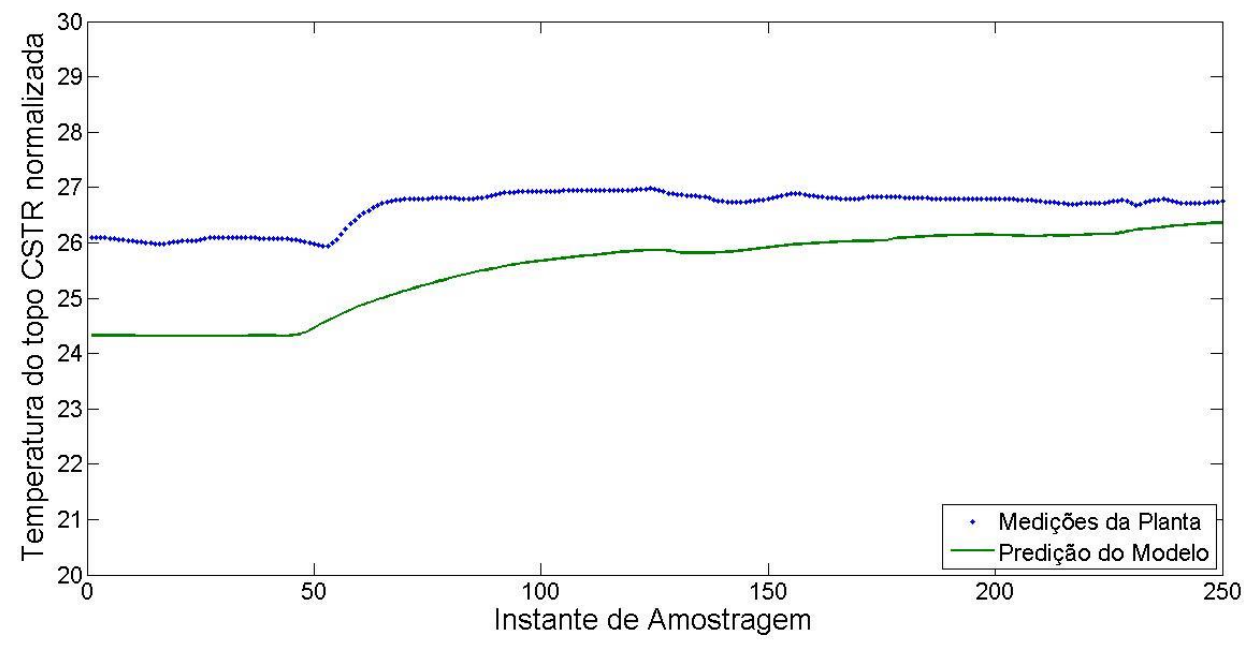

Figura 4 - Valores preditos e experimentais: Temperatura do topo do CSTR normalizada.

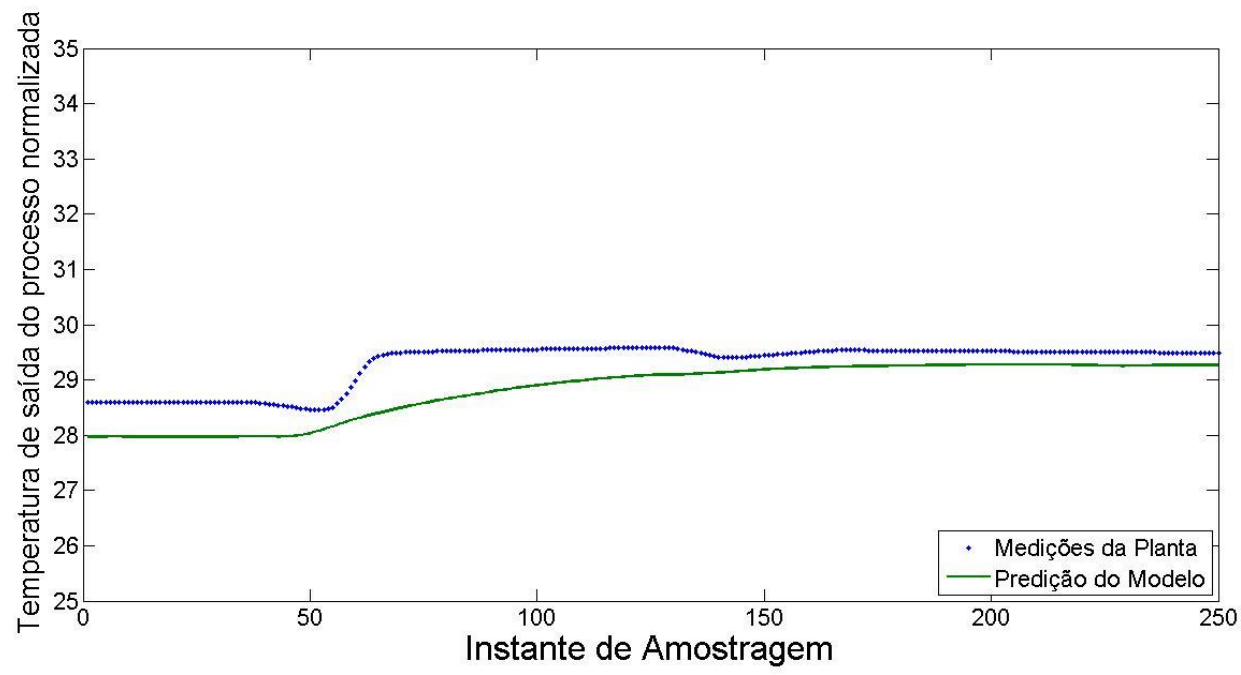

Figura 5 - Valores preditos e experimentais: Temperatura de saída do processo normalizada.

A Figura 6 mostra o mapeamento da função objetivo para alguns pares de parâmetros. Nota-se que a solução do problema por PSO permite a fácil obtenção dos gráficos já que os resultados são calculados ao longo das iterações do algoritmo. Os mapas na região superior da Figura 6 mostram a mesma configuração que a maioria dos outros mapas: uma grande dispersão dos pontos por boa parte do espaço de busca. Já os mapas relativos ao parâmetro 02, ilustrado na região inferior da Figura 6, são muito semelhantes entre si. Os perfis de "cascata" encerrando em um "platô" são evidência de que a influência do parâmetro 02 é muito superior a dos outros. Este parâmetro 02 representa a constante $\mathrm{a}_{0}$ (termo independente da temperatura) da expressão de ajuste polinomial do Cp do etileno (PONTES et al., 2010). 

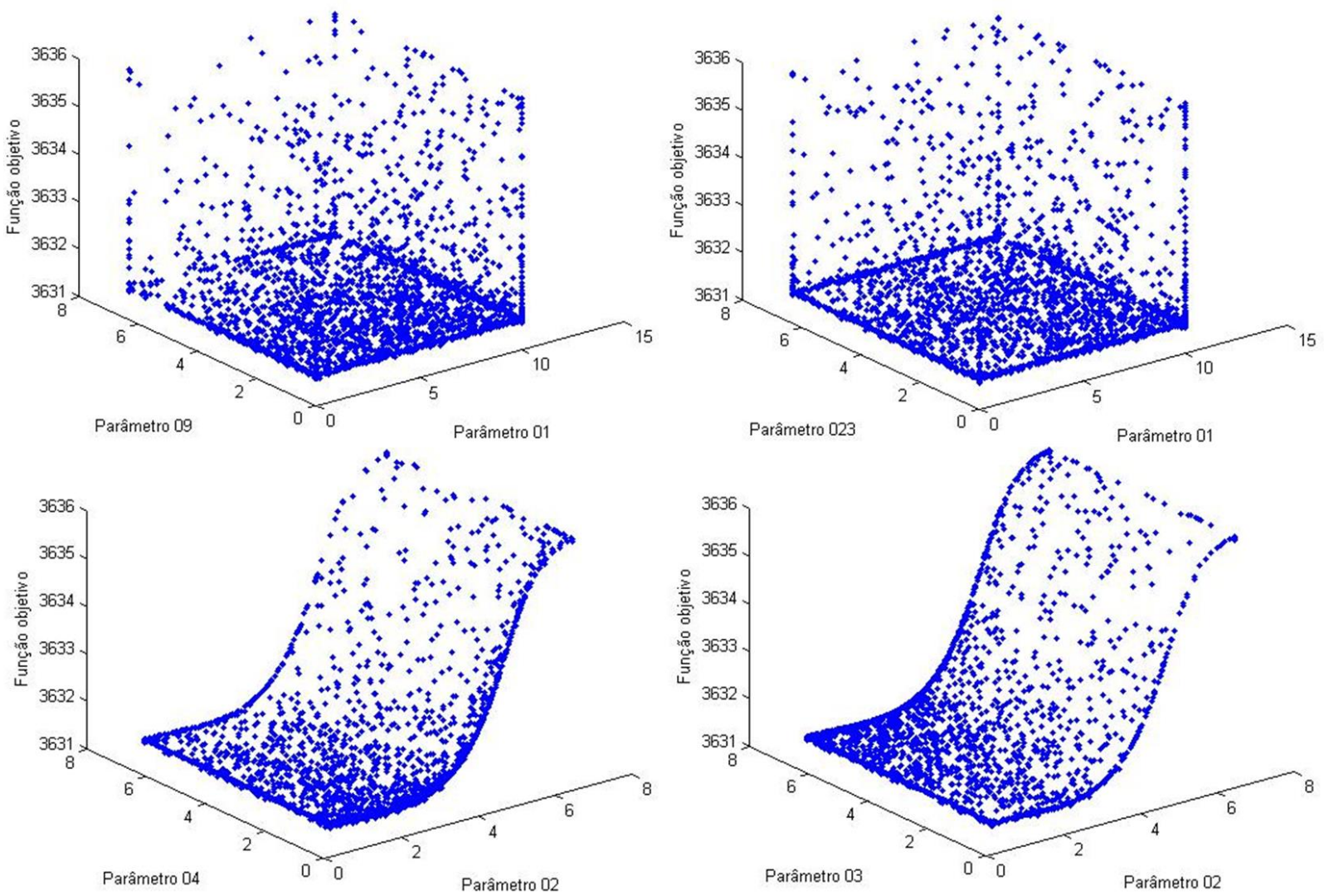

Figura 6 - Mapeamento da função objetivo.

\section{CONCLUSÃO}

Os resultados da estimação e validação mostram que o PSO foi capaz de obter uma boa estimativa dos parâmetros, a despeito da complexidade do modelo, tendo em vista os baixos desvios na validação. Percebe-se também que a influência da constante a de ajuste polinomial do $\mathrm{Cp}$ do etileno é muito superior a de todos os outros parâmetros. Trabalhos futuros devem concentrar-se na geração de regiões de confiança dos parâmetros utilizando o potencial do PSO para tanto.

\section{REFERENCIAS}

BENYAHIA, B. et al. Emulsion copolymerization of styrene and butyl acrylate in the presence of a chain transfer agent. Part 1: Modelling and experimentation of batch and fedbatch processes. Chem. Eng. Science, v. 65, p. 850-869, 2010.

BENYAHIA, B. et al. Emulsion copolymerization of styrene and butyl acrylate in the presence of a chain transfer agent. Part 2: Parameters estimability and confidence regions. Chem. Eng. Science, v. 90, p. 110-118, 2013.

BESSA, I.. N. ; PONTES, K. V. Análise de estimabilidade de um modelo fenomenológico do processo de copolimerização do etileno em solução. COBEQ XX, Florianópolis, 2014.

EMBIRUÇU, M.; PRATA, D. M.; LIMA, E. L.; PINTO, J. C. (2008). Continuous Soluble Ziegler-Natta Ethylene Polymerizations in Reactor Trains, 2 - Estimation of Kinetic Parameters from Industrial Data. 
Macromolecular Reaction Engineering., 2: 142-160.

HOLTZ, G. C. C. Traçado automático de envoltórias de esforços em estruturas planas utilizando um algoritmo evolucionário. 2005. 123f. Dissertação (Mestrado em Engenharia Civil) - Departamento de Engenharia Civil, Pontifícia Universidade Católica do Rio de Janeiro, Rio de Janeiro.

KENNEDY, J.; EBERHART, R. Particle Swarm Optimization. Neural Networks, IEEE International Conference on, v. 4, p. 1942-1948, 1995.

PARK, J. A particle swarm optimization for economic dispatch with nonsmooth cost functions. IEEE Trans. Power Syst, v. 20, p. 34-42, 2005.

POLI, R. Analysis of the Publications on the Applications of Particle Swarm Optimisation. J. of Artificial Evolution and Applications, v. 2008, p. 1-10, 2008.

PONTES, K. V. et al. Modeling and Simulation of Ethylene and 1-Butene Copolymerization in Solution with a ZieglerNatta Catalyst. Int. J. of Chem. Reactor Eng., v. 8, p. A7, 2010.

RATNAWEERA, A. et al. Self-organizing hierarchical particle swarm optimizer with time-varying acceleration coefficients. Evolutionary Computation, IEEE Trans. on., v. 8, p.240-255, 2004.

SCHWAAB, M. Avaliação de algoritmos heurísticos de otimização em problemas de estimação de parâmetros. 2005. 149f. Dissertação (Mestrado em Engenharia Química) - COPPE, Universidade Federal do Rio de Janeiro, Rio de Janeiro.

SCHWAAB, M. et al. Nonlinear parameter estimation through particle swarm optimization. Chem. Eng. Science, v. 63, p. 1542-1552, 2008.

SCHWAAB, M.; PINTO, J. C. Análise de Dados Experimentais I: Fundamentos de Estatística e Estimação de Parâmetros. Rio de Janeiro: Editora E-papers Serviços Editoriais Ltda., 2007.

SHI, Y.; EBERHART, R. A modified particle swarm optimizer. Evolutionary Computation Proceedings, IEEE World Congress on Computational Intelligence, p. 69-73, 1998. 